\title{
Histocompatibility Alloantigens
}

\section{in Psoriasis and Psoriatic Arthritis}

\author{
EVIDENCE FOR THE INFLUENCE OF MULTIPLE GENES \\ IN THE MAJOR HISTOCOMPATIBILITY COMPLEX
}

\author{
C. Murray, D. L. Mann, L. N. Gerber, W. Barth, S. Perlmann, J. L. Decker, and \\ T. P. NigRA, Immunology Branch, National Cancer Institute, Rehabilitation \\ Department of the Clinical Center, Arthritis and Rheumatism Branch of the \\ National Institute of Arthritis, Metabolism and Digestive Diseases, National \\ Institutes of Health, Bethesda, Maryland 20205; Washington Hospital Center, \\ Washington, D. C. 20010
}

\begin{abstract}
A B S T R A C T The frequency of HLA-A, B, and Cw antigens as well as the antigens expressed preferentially on B cells and monocytes (DRw and Ia-like) was examined in a normal population and two related disease populations, psoriasis and psoriatic arthritis. HLA antigens distinguishing the two disease populations were found. Psoriatic patients demonstrated an increase in frequency of HLA-A1, B17, and B13. Patients with psoriatic arthritis demonstrated an increased frequency of HLA-A26, B38, and DRw4. Antigens showing a common increase in frequency in the two disease populations were HLA-Cw6, DRw7, and Ia744. These results demonstrate genetic differences as well as similarities in the two populations of patients with the common clinical feature of psoriasis. In addition to the above analysis, we examined the association of individual alloantigens elevated in frequency in the diseased population. These same alloantigens were examined for association in the normal population. This analysis revealed HLA antigen associations in the two disease groups that differed from the association of several antigens in the normal population. The results suggest that at least two genetic factors, one mapping in the HLA-A, C-B region and one mapping in the HLA-B-DRw region are associated with the disease states. Thus, multiple factors controlled by genes
\end{abstract}

Dr. C. Murray is a Research Fellow of the Department of Dermatology at George Washington University, Washington, D. C., and is in receipt of a grant from the Washington Hospital Center Research Foundation, Washington, D. C.

Received for publication 20 December 1979 and in revised form 27 May 1980. in the major histocompatibility complex appear to contribute to the disease entities under investigation.

\section{INTRODUCTION}

Psoriasis effects $\sim 4 \%$ of the population of the United States (1). This disease of unknown etiology is characterized by increased turnover of epidermal cells and defective keratinization $(2,3)$. Psoriasis may be accompanied by inflammation of peripheral joints and in some cases there is demonstrable axial arthritis. The relationship between psoriasis and psoriatic arthritis is poorly understood. Psoriatic arthritis was thought by some to represent the coexistence of two fairly common diseases, psoriasis and rheumatoid arthritis (4). In the majority of the cases of psoriatic arthritis, the presence of rheumatoid factor cannot be demonstrated. This observation contributes to the recognition of psoriatic arthritis as a separate disease entity (5).

Many external and internal factors have been proposed that may influence the development of the disease in genetically predisposed individuals (6). The genetic predisposition to psoriasis has been the subject of several studies $(7,8)$. It is now well established that genetic factors are implicated in the etiology of psoriasis; however, the mode of inheritance of the genes influencing this disease has not been firmly established. Two possible mechanisms have been proposed. A mendelian dominant inheritance pattern with limited penetrance of the disease susceptibility gene is one hypothesis (7). The other proposes a mechanism with multifactorial genetic control (8).

The association of HLA antigens and certain disease 
groups has provided workers with a potential genetic marker for disease association (9). HLA antigens are gene products of the major histocompatibility complex (MHC), ${ }^{1}$ which is located on the sixth chromosome. Antigens controlled by HLA-A, B, and C loci are serologically defined in cytotoxicity assays, and the D locus is detected by a mixed lymphocyte reaction. It has been reported that the HLA-B13, $\mathrm{B} 17, \mathrm{~B} 37, \mathrm{Bw} 16, \mathrm{Cw} 3$, and Cw6 have increased frequencies in psoriasis (10-19).

Within the past several years another locus controlled by the genes of the MHC has been described. This locus controls the expression of antigens preferentially expressed on monocytes and B cells that are detected by serologic reactions and designated DRw. With the development of antisera-detecting alleles at the DRw locus it is now possible to examine the frequency of these antigens in a variety of disease states.

In the development of reagents detecting antigens on B lymphocytes and monocytes we have observed serologic reactions with these subpopulations of cells that have low frequency of correlation with DRw antigens. It is our opinion that these sera detect other B cell antigens different from the DRw antigens and may constitute gene products of a separate locus in the human MHC (20). We have designated these as Ia-like antigens.

The purpose of this study was to extend previous investigations of the association of HLA alloantigens with psoriasis and psoriatic arthritis and, in particular, to determine whether an increased frequency of particular DRw or other B cell (Ia-like) antigens were present in these two disease populations. Documented herein are the results of our findings. The increased frequency of certain HLA alloantigens, including DRw and Ia-like determinants, demonstrate that psoriasis and psoriatic arthritis are genetically distinct diseases. However, there are common antigens that appear to be increased in frequency in both diseases. Because it was observed that more than one antigen was increased in frequency in the disease, we have examined the possibility that the disease predisposition may be controlled by genetic factors associated with or linked to more than one HLA alloantigen. Our data support the hypothesis that multiple genetic factors controlled by the human MHC have an important influence in psoriasis and psoriatic arthritis.

\section{METHODS}

Patient and control population. Two patient groups were studied. One group consisted of 60 unrelated caucasian patients with psoriasis with $>10 \%$ body surface involvement. None of these patients had any clinical manifestations of arthritis. All forms of psoriasis were represented in the pa-

${ }^{1}$ Abbreviations used in this paper: MHC, major histocompatibility complex. tient group except pustular psoriasis. The other patient group consisted of 52 unrelated caucasian patients with psoriatic arthritis, according to the criteria of Moll and Wright (6).

All patients were diagnosed as having psoriasis by a dermatologist (T.P.N.), and all patients were examined for psoriatic arthritis by several rheumatologists (L.G., W.B., S.P.). The age of the uncomplicated psoriasis patients ranged from 16 to 64 $\mathrm{yr}$ and the psoriatic arthritis group from 17 to $62 \mathrm{yr}$. All patients with psoriatic arthritis as manifested by clinical evidence of joint inflammation were negative for rheumatoid factor. The normal control population consisted of 126 caucasian bloodbank donors with no psoriasis or psoriatic arthritis as determined by questionnaire.

HLA-A, B, C, and DRw alloantigens were determined on peripheral blood lymphocytes by a cytotoxicity method as described by Amos and Pool (21). The alloantisera used in this assay procedure detected 17 alloantigens as controlled by the A locus, 28 alloantigens as controlled by the B locus, and 6 alloantigens as controlled by the $C$ locus. The $\mathrm{DRw}$ alloantigens were detected on $\mathrm{B}$ lymphocytes prepared by the separation technique previously described (22, 23). In brief, this technique is based on the adherence of surface immunoglobulin-bearing cells to plastic plates coated with goat antibody to human IgG F $\left(\mathrm{ab}^{\prime}\right)_{2}$. Whole blood lymphocytes were prepared from heparinized peripheral blood by sedimentation on a Ficoll-Hypaque gradient (lymphocyte separation medium; Litton Bionetics, Inc., Rockville, Md.). B lymphocytes were removed from the whole lymphocyte preparation by adherence to the antiimmunoglobulin-coated plates. The nonadherent cells were removed from the adherent population and the latter population competitively removed with a solution of human immunoglobulin containing $0.5 \%$ EDTA. The recovered cells were found to be $>90 \%$ immunoglobulin-bearing cells, as determined by fluorescence procedures with a fluoresceinated rabbit anti-human immunoglobulin. Alloantisera used to detect the B cell antigens were obtained from multiparous women. To remove HLA activity, the sera were absorbed with 3 vol of packed platelets pooled from at least 60 donors. These sera were tested on a panel of $B$ and $T$ cells to determine the specificity of reaction with a subpopulation of cells.

HLA-DRw and B lymphocyte alloantisera. The patterns of reactions of an individual serum or combinations of sera resulted in the designation of a particular DRw alloantigen as assigned by the Seventh International Histocompatibility Workshop. (24) A number of the sera used in these studies were gifts from investigators in the United States and Europe. Some of the sera used in this study demonstrate reactivity specific for B cells, but have no definitive correlation in their reaction patterns with the assigned DRw specificities. Sera were tested in a complement-dependent cytotoxicity assay as previously described $(22,23)$. Duplicate tests were performed on each individual for determining DRw and Ia-like alloantigens.

Statistical methods. The data were analyzed by comparing the frequency of the HLA-A, B, C, and DRw antigens in the two patient groups with the normal controls. Chi-square test using the Fisher exact test (2-tailed) with Yates correction was used to determine the statistical significance of comparative frequencies of alloantigens.

\section{RESULTS}

Lymphocyte typing in psoriasis. The male/female ratio in the psoriasis population was $2.3: 1$, in the psoriatic arthritis population 1.8:1, and in the control population 1.8:1. No differences in distribution of any of the HLA alloantigenic specificities was found in 
either the disease or normal population on the basis of sex. Table I compares the frequency of occurrence of the HLA-A, B, and C antigens, which appeared altered in the disease groups, with the frequency of those antigens in the normal population. The frequency of occurrence of all of the DRw specificities tested are included in this table. Significant increases were noted with HLA-A1, B13, B17, and Cw6 in the psoriasis population. DRw7 appeared in $23.8 \%$ of the normal population and $58.33 \%$ of the population of individuals having psoriasis. This difference is statistically significant.

In the psoriatic arthritic population the HLA-A26, $\mathrm{B} 38$, and $\mathrm{Cw} 6$ antigens were increased in frequency compared with the normal population. The most marked increase in frequency of DRw specificities was the DRw4 antigen. This antigen was found in $35.7 \%$ of the normal population and increased in frequency to $59.6 \%$ in the population with psoriatic arthritis. DRw7 was modestly elevated from $23.8 \%$ in the normal population to $42.3 \%$ in the psoriatic arthritic group. In both patient populations, a marked decrease in the frequency of HLA-A3, B7, and DRw2 was observed. All

TABLE I

Frequency of HLA-A, B, C, and DRu Antigens in Psoriasis and Psoriatic Arthritis*

\begin{tabular}{|c|c|c|c|}
\hline Antigen & $\begin{array}{c}\text { Normal } \\
(n=126)\end{array}$ & $\begin{array}{l}\text { Psoriasis } \\
(n=60)\end{array}$ & $\begin{array}{l}\text { Psoriatic } \\
\text { arthritis } \\
(n=52)\end{array}$ \\
\hline & $\%$ & $\%$ & $\%$ \\
\hline \multicolumn{4}{|l|}{ HLA } \\
\hline A 1 & 25.39 & $45.00 \neq$ & 30.77 \\
\hline A 3 & 34.13 & $10.17 \S$ & $16.92 \ddagger$ \\
\hline A 26 & 6.35 & 1.67 & $19.23 \ddagger$ \\
\hline B 7 & .30 .95 & $8.33 \S$ & $9.62 \ddagger$ \\
\hline B 13 & 3.89 & $15.00 \S$ & 11.54 \\
\hline B 17 & 7.86 & $38.33^{\prime \prime}$ & 17.31 \\
\hline B 38 & 3.97 & 6.8 .3 & $23.07 \S$ \\
\hline Cw 6 & 13.49 & $50.00^{\prime \prime}$ & $34.61 \S$ \\
\hline \multicolumn{4}{|l|}{ DRW } \\
\hline 1 & 9.52 & 8.33 & 3.3 .3 \\
\hline 2 & 36.51 & $10.10 \S$ & $5.77 \S$ \\
\hline 3 & 26.98 & 21.67 & 23.07 \\
\hline 4 & 35.71 & 31.67 & $59.61 t$ \\
\hline 5 & 17.46 & 8.33 & 42.30 \\
\hline 6 & 34.92 & 41.66 & 34.61 \\
\hline 7 & 23.81 & $58.33^{\prime \prime}$ & 42.30 \\
\hline Ia 744 & 27.78 & $68.33^{\prime \prime}$ & $71.15^{\prime \prime}$ \\
\hline
\end{tabular}

Significance levels determined by statistical methods described in text.

* Only those HLA-A, B, and Cw antigens with significant changes in the diseased populations are listed. All of the DRw antigens tested for are listed.

$\ddagger P \leq 0.05$.

$\S P \leq 0.01$

" $P \leq 0.001$.
TABLE II

HLA-A, B, C Antigens Associated with Psoriasis and Psoriatic Arthritis.

\begin{tabular}{lccccc}
\hline & HI.A-A & HLA-B & HLA-Cw & DRw & Iat \\
\hline $\begin{array}{l}\text { Psoriasis } \\
\begin{array}{l}\text { Psoriatic } \\
\text { arthritis }\end{array}\end{array}$ & 1 & 17,13 & 6 & 7 & 744 \\
\hline
\end{tabular}

of the antisera-detecting $B$ cell alloantigens were analyzed for frequency of reaction in the normal and disease groups. One antisera $\mathrm{Ia} 744$ reacted with $27.7 \%$ of the normal, $68.3 \%$ of the psoriasis, and $71.15 \%$ of the psoriatic arthritis population. Table II summarizes the altered HLA profiles in the two disease groups. HLA-A1, B13, B17, and DRw7 appear to be elevated in patients with psoriasis, and HLA-A26, B.38, and DRw4 are elevated in patients with psoriatic arthritis. Elevations of the HLA-Cw6, and Ia744 antigens were shared in both disease groups.

Two of the HLA-A and B antigens commonly found in linkage disequilibrium were observed to be decreased in the patient population. HLA-A 3 and B7 were found together in $21 \%$ of the normal population, $6 \%$ of the psoriatic arthritis group, and $0 \%$ of the patients with psoriasis. Several of the DRw antigens were also found to be decreased in frequency in the patient groups. The decrease in HLA-DRw2 reached statistical significance. DRw5 was also found to be lower; however, this decrease was not statistically different from the normal population.

HLA alloantigen associations. The elevation in frequency of a number of HLA alloantigenic determinants in these disease groups raised the question as to whether these antigens were elevated independently. The phenomenon of linkage disequilibrium is commonly observed with a number of the HLA alloantigen specificities. If a particular HLA alloantigen is elevated in a population, the increase observed may be because a gene controlling one alloantigen is in linkage disequilibrium with a gene controlling another alloantigen. An elevation of multiple antigens not in linkage disequilibrium would suggest that independent factors controlled by genes of the MHC contribute to the pathogenesis of the disease. An analysis was performed comparing the frequency of association of antigenic determinants in the normal population that appeared elevated in both the disease populations. The results of this analysis are shown in Table III.

This table gives the chi-square and significant $P$ values obtained by this analysis. In the normal population, $\mathrm{Cw} 6$ was associated with $\mathrm{B} 13$ and $\mathrm{B} 17$. B13 was associated with the serologic reactivity of Ia744 to B cells. The Ia744 reactivity is correlated with occurrence 
TABLE III

Analysis of the Association of the HLA Antigens in the Normal Population and Diseased Populations*

\begin{tabular}{lcc}
\hline & Chi square & $P$ value \\
\hline Normal & & \\
Cw6-B17 & 21.182 & $<0.00$ \\
Cw6-B13 & 33.091 & $<0.00$ \\
B13-Ia744 & 6.44 & 0.01 \\
Ia744-DRw4 & 13.42 & $<0.001$ \\
Psoriasis & & \\
Al-B13 & 6.369 & 0.02 \\
Al-Cw6 & 5.498 & 0.01 \\
Cw6-B17 & 8.188 & $<0.01$ \\
Cw6-B13 & 4.101 & 0.02 \\
B13-DRw7 & 7.568 & $<0.01$ \\
Psoriatic arthritis & & \\
A26-B38 & 10.906 & 0.001 \\
DRw7-IA744 & 4.396 & 0.02 \\
\hline
\end{tabular}

* HLA alloantigens elevated in the disease groups were compared for association in the diseased and normal population using $2 \times 2$ contingency tables, Fisher exact test (two-tailed) with Yates correction.

of DRw4. In the psoriatic population, the Cw6-B17, Cw6-B13 associations are maintained, although to a lesser degree. New associations of antigens are now found in this patient population. HLA-Al appears to be associated with Cw6 and B13. In addition, Bl3 now appears to be associated with DRw7. The association of B13 with Ia744 no longer appears evident. The Ia744 reactivity does not appear to associate with any particular HLA alloantigen. This suggests that the serum is detecting an independent antigenic determinant. In the patient population with psoriatic arthritis, DRw7 and Ia744 appear together with increased frequency. The high degree of association of DRw4 and Ia744 evident in the normal population no longer appears associated in this disease group, even though these antigens are both elevated in the disease population. HLA-A26-B38 were found to be associated in this patient population. The HLA-A26-B38 and HLA-B38DRw4 antigen associations have been reported to be $11 \%$ (for each combination) in a nondisease Jewish population of 10 . Because we observed a significant association of the A26-B38 antigens in the psoriatic arthritis population, the frequency of this combination was determined in the Jewish and non-Jewish patient group. The HLA-A26-B38 combination was found in $20 \%$ of the 15 Jewish psoriatic arthritis patients and $10 \%$ of the 32 non-Jewish patients. This combination did not occur in 8 Jewish patients with psoriasis. One individual in the control population (126) typed for HLAA26, B38, and DRw4. The ethnic origin of this individual could not be determined. The HLA-B38-DRw4 combination was found in $26 \%$ of the Jewish patients with psoriatic arthritis and $16 \%$ of the non-Jewish patients. Two of the Jewish patients with psoriasis had this combination of antigens. This HLA-B38-DRw4 association was not increased in frequency in either patient group.

\section{DISCUSSION}

In this report, we have demonstrated significant elevations of the frequency of HLA-A 1, B13, B17, and DRw7, and the serologic reactivity of sera Ia744 in patients with psoriasis. The results support observations previously reported on HLA-Al and Bl7 in this disease (11-19). HLA-Al was found to be part of the "psoriasis haplotype" in family studies $(25,26)$. HLA-Al was also found to be significantly elevated in a Japanese study of psoriasis patients (27). Our finding of an elevation of the HLA-DRw 7 specificity may be comparable with the observation that a significant elevation in the frequency of reaction of a serum defining the LD107 specificity in psoriasis patients was reported at the Seventh International Histocompatibility Workshop (28). Japanese (29) and Israeli (30) studies demonstrated a significant elevation of the HLA-B.37 antigen. This elevation has also been reported in some European family studies $(25,31)$. We did not find a significant elevation of this antigen in our study. This may be the result of the differences in racial and ethnic origin of the patient populations studied. In the patient group with psoriatic arthritis, elevations in frequency of the HLA alloantigens A26, B.38, Cw6, DRw7, and DRw4 were observed. HLA-B38 was reported to be elevated in two previous studies $(32,33)$. This report documents the first study of $\mathrm{Cw}$ and DRw locus antigen frequencies in psoriatic arthritic patients.

It is quite clear from our studies that the HLA profiles of the two patient groups show significant differences; however, there are common antigenic features to both groups. The most outstanding similarities are in the frequency of the Cw6 and Ia744 antigens. The elevation of these antigens in both diseases suggests a significant marker(s), perhaps for the skin lesion itself, as this is the common component of the two disease groups. The other common feature was the elevation of DRw7. This was found to be significantly elevated in the patient population with psoriasis. An elevation in frequency of this antigen was observed in the patient group with psoriatic arthritis; however, this was not statistically significant. In further analysis of this patient group, DRw7 is increased in frequency in patients with mild arthritis and decreased in frequency in patients with severe arthritis (umpublished observation).

The elevation of DRw4 in the arthritic group may represent a contribution of genetic factors, closely linked to genes controlling the expression of this anti- 
gen, to the disease component. DRw4 has been reported to be significantly elevated in adult rheumatoid arthritis (34). In addition, this antigen has been shown to be elevated in frequency in patients with sicca syndrome who have clinical manifestations of arthritis (35).

The elevation of several of the HLA antigens in the disease population may be the result of linkage disequilibrium. The $\mathrm{Cw} 6, \mathrm{~B} 13, \mathrm{~B} 17$ association found in the normal population is maintained (although to a lesser degree) in the disease population. Thus, the increased frequency of the $C$ locus antigen may be the result of the increased frequency of the B locus antigen or vice versa. It is our interpretation that the elevation of the B locus antigens observed in the psoriasis population is the result of the increased frequency of the $\mathrm{Cw} 6$ antigens. In the related disease, psoriatic arthritis, the Cw6 increase is independent of other HLA alloantigens. The elevation in frequency of $\mathrm{Cw} 6$ in both disease groups suggest a common contributing genetic factor.

Several antigens were found to be associated in the disease groups, whereas these same associations were not observed in the normal population. The HLA-Al antigen appears to be associated with $\mathrm{Cw} 6$ in the psoriasis patients, an association not found in the normal population. HLA-A26, B38 association was observed in the psoriatic arthritis group. The fact that an A locus antigen is associated with a $\mathrm{C}$ or $\mathrm{B}$ locus antigen in both disease populations suggests a genetic contribution of the portion of the MHC that maps between $\mathrm{A}, \mathrm{C}$, and B. There also appears to be an additional genetic factor(s) that influences the disease condition dependent on the portion of the MHC controling the DRw-Ia-like alloantigens. It was observed that the Ia744 antigen, increased in frequency in both disease groups, occurs in association with the DRw4 and B13 antigens in the normal population. These associations change in the disease groups. In the psoriasis population, Ia744 does not appear to be associated with any other antigen, and in the psoriatic arthritis group the antigen is associated with DRw7. It is likely, therefore, that the gene coding for the Ia744 antigen is different from the DRw gene but maps in the region of HLA-B-DRw. This contention is supported by analysis of the molecular weight of the cell surface structures reacting with these sera. Sera Ia744 precipitates molecules with molecular weights of 31,000 and 29,000, whereas antiDRw4 sera precipitates molecules with molecular weights of 34,000 and 28,000 (Mann, D. L., and Strominger, L., unpublished observation).

The elevation in frequencies of several HLA alloantigens and the altered association of these antigens in the disease population suggest a multifactorial genetic influence in the disease population studied. The biologic importance of the observed differences remains to be determined. However, the results sug- gest that gene interaction may play a role in disease manifestation. Because we are examining phenotype frequencies, the nature of the gene interaction cannot be discerned from this study. Genes associated with or linked to those genes controlling the expression of the particular antigens or antigen combinations observed to be elevated in frequency may interact in a cis (same haplotype) or trans (cross haplotype) configuration for the pathophysiologic mechanisms to occur, which result in the manifestation of the disease. Family studies, wherein more than one member of a family demonstrates a particular disease component, are needed to resolve this question.

\section{ACKNOWLEDGMENTS}

The authors wish to thank Drs. Howard Dickler and Alfred Singer for their helpful suggestions. We would also like to acknowledge the technical assistance of Linda Pelleu and the assistance of the secretarial support staff. HLA typing was performed on contract NCI-CB-53890.

\section{REFERENCES}

1. Thomas, R. J. 1977. Psoriasis case rate-HL-A antigen frequency correlations. Dermatologica (Basel). 154: 23-31.

2. Voorshees, J. J., E. A. Duell, M. Stawiski, and E. R. Harrell. 1974. Adv. Cyclic Nucleotide Res. 4: 117-162.

3. Weinstein, G. D., and P. Frost. 1971. Methotrexate for psoriasis: a new therapeutic schedule. Arch. Dermatol. 103: $33-38$.

4. Schamberg, J. F. 1924. The known and the unknown about psoriasis. JAMA (J. Am. Med. Assoc.). 83: 1211 (Abstr.)

5. Moll, J. M. H., and V. Wright. 1973. Psoriatic arthritis. Semin. Arthritis Rheum. 3: 55-78.

6. Farber, E. M., and A. J. Cox, editors. 1971. Psoriasis. Stanford University Press, Stanford, Calif.

7. Kimberling, W., and R. L. Dobson. 1973. The inheritance of psoriasis. J. Invest. Dermatol. 60: 528-540.

8. Watson, W., H. M. Cann, E. M. Farber, and M. L. Nall. 1972. The genetics of psoriasis. Arch. Dermatol. 105: 197-207.

9. Dausset, J., and A. Svejgaard, editors. 1977. HLA and Disease. Munksgaard, Copenhagen, Denmark.

10. Park, M. S., P. I. Terasake, A. R. Ahmed, and J. L. Tiwari. 1979. HLA-DRw in $91 \%$ of pemphigus vulgaris patients. Lancet. II: 441-442.

11. Russel, J. T., M. L. Schultes, and D. J. Kuban. 1972. Histocompatibility (HL-A) antigens associated with psoriasis. N. Engl. J. Med. 287: 738-740.

12. White, H. S., V. D. Newcomer, M. R. Mickey, and P. I. Terasaki. 1972. Disturbance of HL-A antigen frequency in psoriasis. N. Engl. J. Med. 287: 740-743.

13. Krain, L. S., V. D. Newcomer, and P. I. Terasaki. 1973. HLA antigens in psoriasis. N. Engl. J. Med. 288: 1245. (Abstr.)

14. Seignalet, J., J. Clot, J. J. Guilhou, F. Duntze, J. Meynadier, and M. Robinet-Levy. 1974. HL-A antigens and some immunological parameters in psoriasis. Tissue Antigens. 4: 59-68.

15. Svejgaard, A., L. Staub Nielsen, E. Svejgaard, F. Kiss- 
mever-Nielson, A. Hjorksh $\phi$ j, and H. Zachariae. 1974. HL-A in psoriasis and in vulgaris psoriasis-population and family studies. Br. J. Dermatol. 91: 145-153.

16. Marusson, J., E. Moller, and N. Thyresson. 1975. HLA antigens $(17,27$, UPS) in psoriasis with special reference to patients with arthritic lesions. Acta Dermato-Venereol. 55: 2.97-300.

17. Woodrow, J. C., V. K. Dave, N. Usher, and J. Andersson. 1975. The HL-A system and psoriasis. Br. J. Dermatol. 92: 427-436.

18. Beckman, L., K. Bergdahl, B. Cerdergren, and S. Liden. 1977. Genetic markers in psoriasis. Correlation to age of onset, continuity of symptoms and the risk of developing the disease. Acta Dermato-Venereol. 57: 247-251.

19. Mc.Michael, A. J., V. Morhenn, K. Payne, T. Susazuki, and E. M. Farber. 1978. HLA C and D antigens associated with psoriasis. Br. J. Dermatol. 98: 287-292.

20. Mann, D. L., J. Kaufman, H. Orr, R. Robb, and J. Strominger. 1979. Serologic and structural studies of DR related antigens. Transplant. Proc. 9: 668-673.

21. Amos, D. B., and P. Pool. 1976. HLA typing in Manual of Clinical Immunology. Rose, N. R., and H. Friedman, editors. American Society for Microbiology. 797-804.

22. Stingl, G., S. Katz, L. D. Abelson, and D. L. Mann. 1978. Immunofluorescent detection of human B cell alloantigens in S-Ig positive lymphocytes and epidemal Langerhans cells. J. Immunol. 120: 661-664.

23. Mann, D. L., L. Abelson, P. Henkart, S. Harris, and D. B. Amos. 1975. Specific human B lymphocyte alloantigens linked to HLA. Proc. Natl. Acad. Sci. U. S. A. 72: 5103-.5106.

24. Bodmer, J. G., P. Pickbourne, and S. Richards. 1978. Ia Serology Histocompatibility Testing. W. F. Bodmer, J. R. Batchelor, and J. G. Bodmer, H. Festenstein, and P. J. Morris, editors. Munksgaard, Copenhagen, Denmark. $38-82$.

25. Marcusson, J., E. Moller, and N. Thyresson. 1976. Penetration of HLA linked psoriasis predisposing gene(s) a family investigation. Acta Dermato-Venereol. 56: 453463.

26. Gunn, I., W. Leheny, T. Lakshmipati, M. A. Lamont, and M. Faed. 1979. HLA antigens in a Scottish psoriatic population. Tissue Antigens. 14: 157-164.

27. Tsuji, K., Y. Nose, M. Ito, A. Ozawa, I. Matsuo, K. Niizuma, and M. Ohkido. 1976. HLA antigens and susceptibility to psoriasis vulgaris in a non-Caucasian population. Tissue Antigens. 8: 29-33.

28. Tiillikainen, A., A. Lassus, R. Pirskanen, P. Vartiainen, and G. Awellán. 1977. An attempt to evaluate Ia type antigens in patients with psoriasis or myasthenia gravis. Tissue Antigens. 10: 203.

29. Ohkido, M., A. Ozawa, I. Matsuo, K. Niizuma, M. Nakamo, K. Tsuji, Y. Nose, M. Ito, T. Sugai, and T. Yasuda. 1976. HLA and Ia antigens and susceptibility to psoriasis vulgaris. 1st International Symposium HLA and Disease. Paris, June 23-25. (Abstr.)

30. Gazit, E., S. Brenner, T. Efler, S. Orgad, Y. Mizradin, and A. Krakowski. 1978. HLA antigens in patients with psoriasis. Tissue Antigens. 12: 195- 199.

31. Karovonen, J. 1975. HLA antigens in psoriasis with special reference to the clinical type, age of onset, exacerbations after respiratory infections and occurrence of arthritis. Ann. Clin. Res. 7: 301-311.

32. Roux, H., P. Mercier, D. Maestracci, G. Serratrice, J. Sanny, J. Seignalet, and H. Serre. 1977. Psoriatic arthritis and HLA antigens. J. Rheumatol. 4: 64-65.

33. Espinoza, L. R., F. B. Vasey, J. H. Oh, R. Wilkinson, and C. K. Osterland. 1978. Association between HLA-B38 and peripheral psoriatic arthritis. Arthritis Rheum. 21: 72-75.

34. Stastny, P. 1978. Association of the B-cell alloantigen DRw in Rheumatoid arthritis. N. Engl. J. Med. 298: $869-871$.

35. Moutsopoulus, H. M., D. L. Mann, A. H. Johnson, and T. M. Chused. 1979. Genetic differences between primary and secondary sicca syndrome. N. Engl. J. Med. 301: 761-763. 